\title{
A New Approach to Ontology-Based Semantic Modelling for Opinion Mining
}

\author{
Rowida Alfrjani, Taha Osman and Georgina Cosma \\ School of Science and Technology \\ Nottingham Trent University \\ Nottingham, UK \\ e-mail: rowida.alfrjani2013@my.ntu.ac.uk, taha.osman@ntu.ac.uk, georgina.cosma@ntu.ac.uk
}

\begin{abstract}
With the fast growth of World Wide Web 2.0, a great number of opinions about a variety of products have been published in blogs, forums, and social networks. Opinion mining tools are needed to enable users to efficiently process a large number of reviews found online, in order to determine the underlying opinions. This paper presents a new methodology for semantic modelling of the domain knowledge for opinion mining. In particular, the new methodology focuses on modelling the domain knowledge in such a way that it can be translated to a formal ontology, which can then be automatically enriched with ground facts obtained from public Linked Open Data resources. The methodology also considers procedures to link between the formal ontology and Natural Language Processing. Our approach successfully enriches the ontology with the relevant ground facts. This ontology can then be used to perform a variety of data mining tasks including sentiment analysis and information retrieval.
\end{abstract}

\section{Keywords - Semantic Modelling; Opinion Mining; Knowledge Base; Ontology}

\section{INTRODUCTION}

With the fast growth of World Wide Web 2.0, a great number of opinions about a variety of products is published in online blogs, forums, and social networks. Opinions play an important role in supporting consumers to make decisions about purchasing products or services. In addition, customer reviews allow companies to understand the strengths and limitations of their products and services and improve upon these. Such valuable information can only be obtained via appropriate analysis of the opinions expressed by those customers who have purchased the products/services and have expressed their experiences in the form of on-line textual reviews. It is important to adopt tools and techniques that can effectively analyse the opinions and allow for qualitative information extraction. Qualitative information can provide an insight into the opinions and allow for information to be retrieved based on searching for emotions and opinions on various product features. An insight into customer emotions about product features can provide a more detailed understanding of the customer reviews, and will facilitate the analysis of the underlying sentiments on product features. Thus, an increasing number of studies about opinion mining have emerged to enhance the end-user search experience.

Opinion mining aims to automatically analyse and determine opinions expressed as natural language text.
Opinion mining is commonly applied by extracting reviews for a specific domain (e.g. movie, music, and product) and performing opinion analysis at various levels of text granularity: document, sentence or feature level. Opinion mining at document and sentence level aims to classify the overall sentiment orientation that is expressed in documents [6] and at sentences [4]. Opinion mining at feature level is especially challenging as it entails extracting domain features, associating each feature with its sentiment, as well as classifying the orientation of a sentiment that describes each feature. Opinion mining at feature level requires a deep understanding of the structure and knowledge of the domain to extract features and their relevant feature sentiments, in order to correctly determine the polarity of each feature.

Opinion mining research at feature level employs different techniques to primarily improve the feature extraction task, which in consequence enhances the performance of feature-sentiment association and feature polarity tasks. Each technique relies on a specific direction for extracting features from reviews. For example, Association Rule Mining algorithms (ARM) primarily rely on natural language processing techniques to identify nouns and noun phrases representing features $[7,8,10,17]$. Machine Learning approaches (ML) rely on a large set of training data to learn the features from a set of reviews $[2,11,19]$. Semantic Knowledge-Based approaches (SKB) are based on extracting features from reviews by utilising an ontology that contains a conceptualised knowledge background of the domain $[1,14,18]$. Several attempts on using the SKB approach to extract features using domain knowledge are discussed in the literature, and these present promising results in comparison with aforementioned ARM and ML approaches [3].

The work presented in this paper investigates the utilisation of Semantic Web in opinion mining and presents an integrative methodology that captures all the phases of semantic modelling including domain knowledge conceptualisation, ontology engineering, natural language processing and sentiment matching.

The remaining of the paper is organised as follows: related work is discussed in section II, Section III describes the process for semantic modelling of the domain knowledge. Section IV discusses in detail how to integrate semantic ontology with natural language process for opinion mining process. A discussion and conclusion are presented in Section V. 


\section{RELATED WORK}

This section discusses related literature in opinion mining with a focus on feature extraction approaches from natural language text consisting of reviews.

$\mathrm{Hu}$ and Liu [10], Eirinaki et al. [7], Ghorashi et al. [8] and Yang et al. [17] applied ARM algorithms that primarily rely on Natural Language Processing (NLP) techniques for feature extraction. ARM algorithms extract features without performing human pre-processing tasks (e.g. preparing manually training dataset) because automatic NLP techniques are used to identify nouns and noun phrases as features. However, the extracted features tend to be frequent features, whereas infrequent features are ignored, which can reduce the recall of feature extraction task. In addition, frequent features can be non-domain's features and this will decrease the accuracy of the extracted features.

Zhuang et al. [19], Ma et al. [11] and Agarwal et al. [2] performed ML approaches that require largely trained datasets to perform with accuracy. ML approaches deliver significant results for feature extraction task when the training data sets are manually annotated by a human expert. However, this can be an extremely time-consuming task as the required size of the training dataset should be sufficiently large to bootstrap the learning algorithms.

Zhao and $\mathrm{Li}$ [18], Peñalver-Martínez et al. [14] and Agarwal et al. [1] utilised SKB approaches that are mainly based on the knowledge background of the domain. SKB approaches demonstrated improved performance for feature extraction when the knowledge of the domain of interest is utilised to extract features.

In this research, we present our methodology for semantic modelling of domain knowledge and for gathering domain-associated data in preparation for further analysis related to the opinion mining process.

\section{A NeW Approach to Semantic Modelling OF THE DOMAIN KNOWLEDGE}

Domain Knowledge is knowledge about a domain's environment, i.e. key concepts and their synonyms and ground facts, as well as the relation between them [5]. Such domain knowledge can be utilised to improve the processes of opinion mining process.

The Semantic Web (SW) is "an extension of the current Web, where information is given a well-defined meaning, encouraging cooperation among human users and computers" [12]. The Semantic Web is concerned with making unstructured data on the Web more understandable to computers via adding linguistic and semantic metadata to the web content.

Semantic Web technologies organise knowledge in formalised concept ontologies that provide efficient support for linking and sharing data between resources, and presenting data in a way that computer machines can process. In addition, a semantic ontology is capable of presenting the domain knowledge in a structured and consistent way which facilitates the qualitative interpretation of domain specific contents in a way that people can understand. Moreover, Semantic Web technologies provide support for enriching the modelled domain knowledge base with relevant ground facts from public-sourced Linked Open Data (LOD) resources. Matthews comments that:

"Semantic Web technologies provide a common framework that allows data to be shared and reused across application, enterprise, and community boundaries. It is a collaborative effort led by W3C with participation from a large number of researchers and industrial partners. It is based on the Resource Description Framework, which integrates a variety of applications using XML for syntax and URIs for naming" [12].

In this section, we elaborate on our methodology for semantically modelling of knowledge for a chosen domain. Firstly, the domain knowledge should be modelled, and then translated into a formal semantic ontology. Finally, the formal domain ontology should be enriched with ground facts extracted from LOD resources.

The case study for our proposed modelling methodology is the movie reviews domain, which can be considered more challenging than modelling other domains such as product reviews. This is because movie reviews tend to include a rich set of the concept (movie), and features (actors, script, plot, etc.) levels. In addition, in movie reviews, users tend to use different words (synonyms) for describing the same feature as well as to express opinions on people who are related to the movie such as "Sally Lee was amazing in this movie". Furthermore, the popularity of the movie domain provides for the opportunity to exploit the ever-increasing crowdsourced LOD repository corresponding to the movie and celebrity industry.

\section{A. Modelling the Domain Knowledge}

Modelling the domain knowledge is based on capturing its knowledge into concepts that are connected together using relations. In addition, the model should illustrate the external relations interrelating concepts from different domains. In our work, the scenario is based on modelling a domain knowledge that can be used in the next stage of our research where opinion mining process will be carried on movie reviews at feature level using SKB approach. Hence, using a concept map tool, we designed a model that connects three domains: Movie, Opinion and Review. The model is called MOR, which is an abbreviation for Movie, Opinion, and Review.

For the Movie domain, our model captures its key concepts such as actor, writer, producer, editor, sound, script, twist, performance, special effect, footage, humour, movie theme, costume, cinematography, emotion, scene, images, ends, background, pacing, staging, story, plot, style and sets as well as their synonyms and the relationships between them.

Regarding the opinion domain, our model presents the expressed opinions with their polarities. In addition, the model presents the relationships between these opinions and the domain's features, that the opinions refer to, as well as the sentiments expressed in them. 


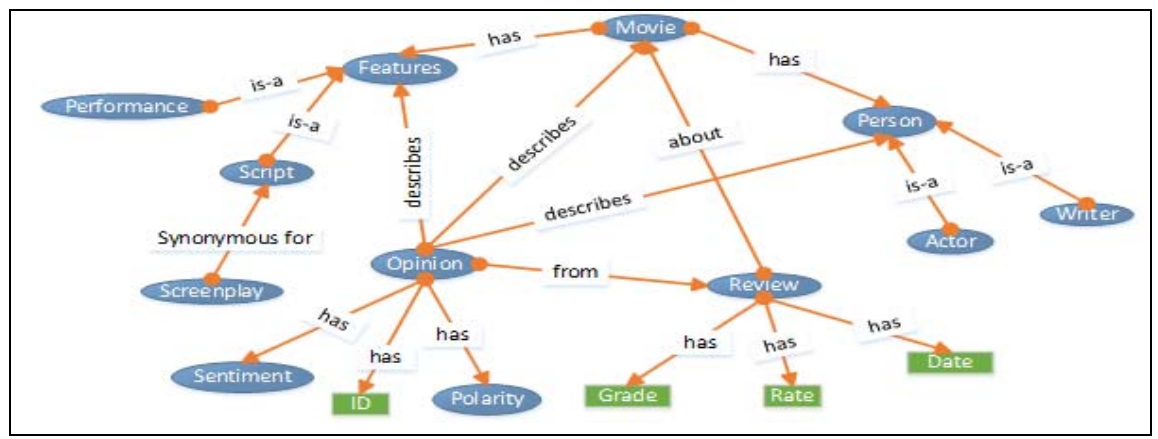

Figure 1: MOR modelled knowledge

For the review domain, the model covers review information that is counted as the source of the extracted opinions about a specific movie.

The knowledge model encompassing the relationships between the three mentioned domains as shown in Fig. 1. Thus, we can absorb and associate generic information about the movie, opinions as well as its reviews. In addition, our model is presented in a structured way that allows it to source data from external LOD datasets set such as DBpedia.

\section{B. Generating a Semantic Ontology}

The MOR modelled knowledge was translated into a formal semantic ontology comprising a comprehensive knowledge of the movie domain as well as opinion and review domains.

For the movie domain, we manually collected a wide range of movie domain key concepts and their synonyms from the Movie Terminology Glossary [13]. Then, we categorised the collected terms as classes, instances, object relationships and synonyms. The created primary classes are Movie, Features and Person. The class Movie is a simple upper class that contains all the individuals that characterise movie names. Each movie has datatype values such as released date and running time. In addition, the class Movie has the annotation synonyms that contain the synonymous words for the concept "movie" such as "film, show and picture", and hence different terms can be mapped to one concept during extracting features from contents for opinion mining process. The classes Person and Feature are upper classes that capture movie domain's key concepts and their synonyms as well. Some of these key concepts such as Star, Writer, Director, Cinematographer, Producer, Editor are subclasses of the class Person, which represent names of people as individuals with respect to their roles in the movie. For example, the class Stars contains names of actors and actresses as individuals. The rest of the key concepts such as Writing, Editing, Special Effect, Animation, Sound, Music, etc. are subclasses of the class Feature. Some of these subclasses are super classes because they contain some subclasses. For example, the class Script is a subclass of the class Writing that captures movies' scripts.

Regarding the opinion domain, we linked our movie ontology with the Marl ontology [9], which is an ontology designed to describe and annotate opinions that expressed by users for a specific domain. The Marl ontology contains two classes named Opinion and Polarity. The Opinion class covers the extracted opinions as well as their relevant information such as Opinion Text and Polarity value.

In addition, the Opinion class contains some relations (described-feature, described-object and extracted-from) which are the keys used for linking our movie ontology with the Marl ontology, since the Marl ontology model is not complete. This made it possible to use the Marl ontology for the task of addressing the extracted opinions from the textual reviews. The Marl ontology:

"mainly defines concepts that are not described yet by the means of other ontologies and provides the data attributes that enable to connect opinions with contextual information already defined in metadata created with other ontologies" [9].

The opinion mining process focuses on extracting sentiment terms that are used to express opinions, and for this reason, the Sentiment class was created later. The Sentiment class includes sentiment terms such as great, bad, good, interesting, etc.

For the review domain, the Review class was created to capture the semantic information about reviews that contain opinions. The Review class contains instances that cover information about reviews such as review number and reviewer name.

Finally, four more upper classes were created: Award, Location, and Language to capture other semantic information about movies such as the nominated award, filming location, and the original language.

The advantage of utilising Semantic Web technologies to represent the domain's key concepts, their synonyms and ground facts, is that all created classes such as Movie, Person, Features, Opinion, Polarity, Sentiment, Review, Award, Country and Language, were linked together using typed object relationships. Examples of such relations are movie has a feature; movie has a person; movie has an award; movie was filmed in a country; review about a movie; opinion extracted from a review; opinion describes a feature; opinion has a polarity; opinion is expressed by a sentiment. This information can be used to infer valuable and strong semantic information about the domain as well as the expressed opinions on its features by: (1) summarising the overall opinion about a movie across multiple reviews, and (2) by summarising overall opinions about other cinematic 
features (actors, script, sound effects, etc.). For example, the movies that have a great screenplay can be retrieved via running one query on our enriched knowledge base.

\section{Enriching the Formal Ontology}

Enriching the ontology with information and facts related to the chosen domain can be conducted manually or automatically. In this work, we enriched the semantic ontology with relevant ground facts that were captured from public movie data sets. Such facts included movie name, actor names, released date, running time, country, and language.

The DBpedia ontology is the best source for collecting such ground facts because it contains richer information about the movie domain than other ontologies. DBpedia ontology is a knowledge base that covers multi-domains and enriched with lots of structured ground facts for each domain. These ground facts are extracted from Wikipedia pages. The DBpedia ontology aims to represent actual community agreement, to be decentralised, to be evolved automatically when Wikipedia changes and to support multilanguages. Moreover, the DBpedia ontology is stored in the Resource Description Framework and it is available on the Web as one of the LOD resources which can be semantically retrieved and manipulated using the SPARQL query language [16]. For example, during the ontology enriching process, movie title is extracted from the review, then the relevant ground facts about this movie (movie's name, released date, running time, country and language; movie's stars, directors, writers, editors, cinematographers and producers) are gathered from DBpedia and Internet Movie Database $(\mathrm{MDb})$ and enriched into the ontology by following the illustrated steps in in Fig. 2. These steps are performed via SPARQL Construct queries, which is an: "RDF query language, that is, a semantic query language for databases, which is able to retrieve and manipulate data stored in Resource Description Framework (RDF) format" [16].

\begin{tabular}{|l}
\hline 1 FOR each Review \\
2 GET Movie's Title as $T$ \\
3 SEARCH for a wiki page about $T$ \\
3.1 IF the Wiki page is found THEN \\
3.1 .1 GET Wiki URI as $W_{-} U R I$ from the obtained page \\
3.1 .2 REPLACE the first part of $W_{-} U R I$ : \\
http://en. wikipedia org with first part of DBpedia URI: \\
http://dbpedia.org/resource as $D_{-} U R I$ \\
3.1.3 INSERT into Ontology Movie's $T$ details via SPARQL \\
Construct Query and $D_{-} U R I$ \\
4 SEARCH for IMDb page about $T$ \\
4.1 $\mathbf{I F}$ the IMDb page is found THEN \\
4.1.1 GET Movie's $T$ Stars \\
4.1.2 INSERT into ontology Movie's $T$ Stars via SPARQL Query \\
5 END FOR \\
Figure 2: Ontology enrichment process
\end{tabular}

Figure 2: Ontology enrichment process
Fig. 3 illustrates a SPARQL Construct query for collecting relevant ground facts for THE ADDICTION_1995 movie.

The consequence of performing the ontology enrichment process is that we end-up with a rich set of semanticallystructured information about each movie including movie name, released date, running time, country, language, stars, directors, writers, editors, cinematographers, producers, etc., which is considered invaluable for the process of opinion mining.

\section{INTEGRATING THE SEMANTICALLY MODELLED ONTOLOGY WITH NATURAL LANGUAGE PROCESSING ENGINE}

In this section, we discuss the process of integrating a semantic ontology for a chosen domain with a natural language processing (NLP) engine in order to utilise it for opinion mining tasks. A snapshot of our Pipeline system is shown in Fig. 4.

\section{A. Natural Language Pre-Processing}

The process of mining domain reviews requires to firstly pre-process each textual review linguistically and syntactically. There are various implemented NLP components that can be adopted for analysing reviews such as the tools that have been implemented via the open source software called GATE (http://gate.ac.uk/). The preprocessing phase is described in below:

1) Tokenisation: each review in the dataset is converted into tokens. Each token has a unique number, position (start and end), and other features such as length of the token.

2) Sentence Splitting: each tokenised review is split into sentences based on a delimiter such as a full stop punctuation mark ".".

3) Part of Speech Tagging: is applied to identify the part of speech (POS) of each token in the review whether it is a noun, verb, adjective, adverb, etc. This category will be

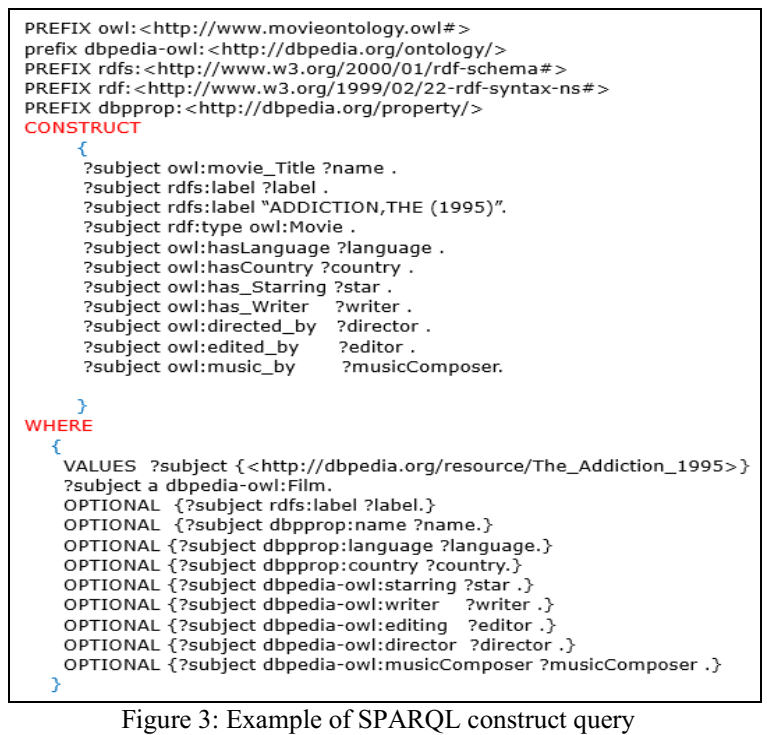




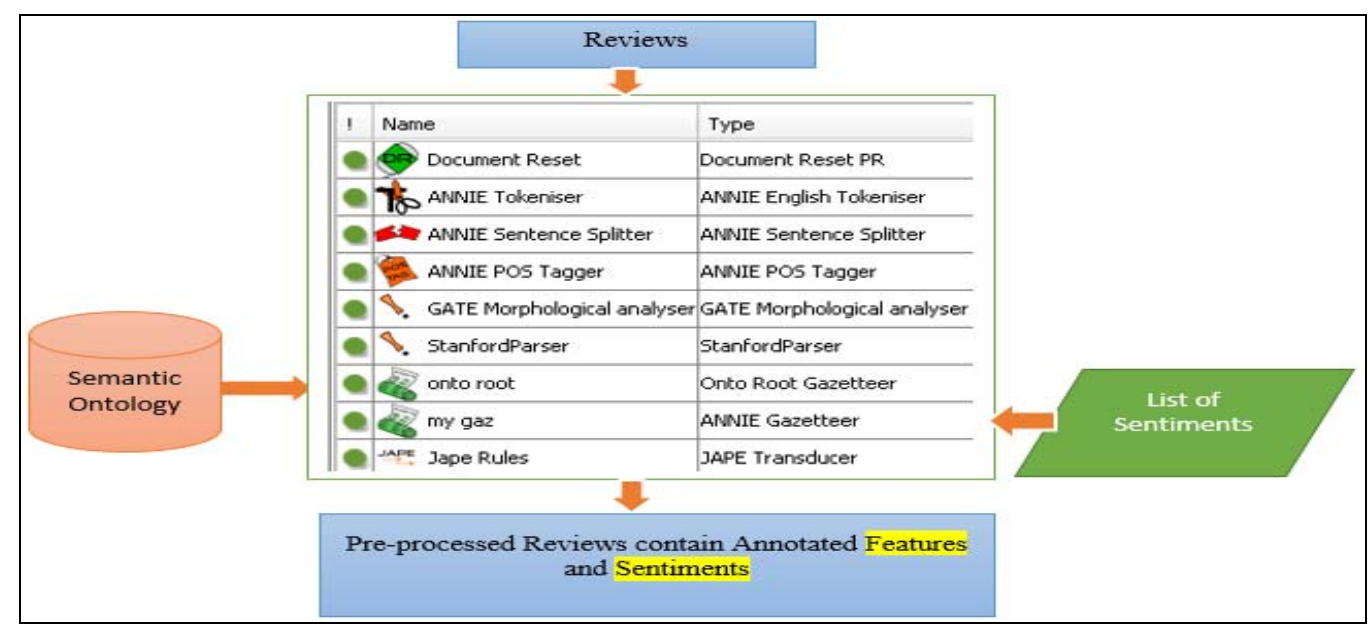

Figure 4: System pipeline for integrating a Semantic Ontology with a Natural Language Processing engine

added to each token as a feature.

4) Morphological Analysis: is about formatting each token in the review to its root. This feature "root" will be added to the token as a feature.

5) Syntax Parsing: aims to identify the grammatical relationships between tokens in a sentence such as "amod" and "nsubj" for adjectival phrase and noun subject phrase respectively.

In our work, tokenisation, sentence splitting, and part of speech tagging, were performed using the relevant components found in A Nearly-New Information Extraction System (ANNIE) that is included within GATE. Regarding morphological analysis, we relied on the GATE Morphological component. Finally, we adopted the Stanford Parser as an embedded application in GATE for syntax parsing. Fig. 5 illustrates the outputs of NLP processing in GATE on the sentence "This movie makes me happy". In the outputs, "Token.Category" points to the POS for each token, and "Token.root" indicates the root of each token. "Dependency.kind" lists various relationships between tokens.

\section{B. Feature Annotation}

Feature annotation or extraction is the second phase of the opinion mining process after pre-processing reviews

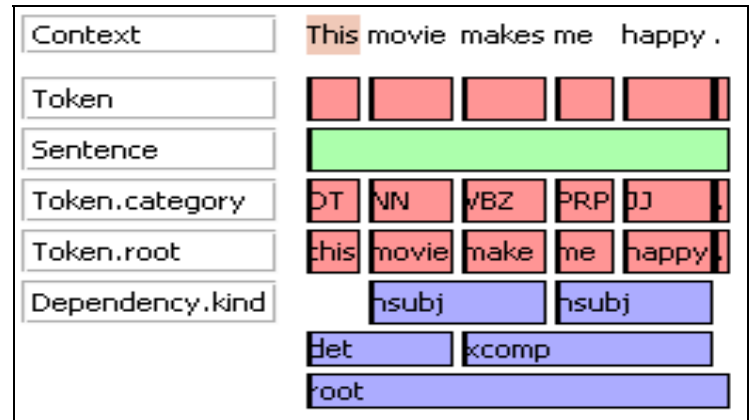

Figure 5: Example of a processed sentence linguistically and syntactically linguistically and syntactically. As mentioned in the related work section, different techniques can be performed to extract a domain's features from reviews. In our work, the feature extraction process is primarily driven by a domain knowledge that is structured in a formal semantic ontology.

In this research, we used GATE's Onto Root Gazetteer (ORG) to link between the root of each word in the preprocessed reviews and our pre-processed ontology. In particular, ORG annotates features (domain's key concepts, synonyms and ground facts) using a flexible and dynamic source of a gazetteer. This gazetteer is produced by ORG in which it pre-processes the ontology by means of tokenisation and morphological analysis. The annotated features within the reviews are given the same classification within the ontology. For example, the annotated word "movie" is classified as a class because it is mapped using ORG to the class Movie in the ontology; whereas, the annotated word "The_Addiction_1995" is classified as an instance of the class Movie, and this also applies to synonyms, attributes and relationships. Fig. 6 presents a snapshot of annotated features by ORG. It is important to mention that ORG annotates all features with their classification under a set called "Lookup", hence we divided the annotated features based on their classification. For example, features that are

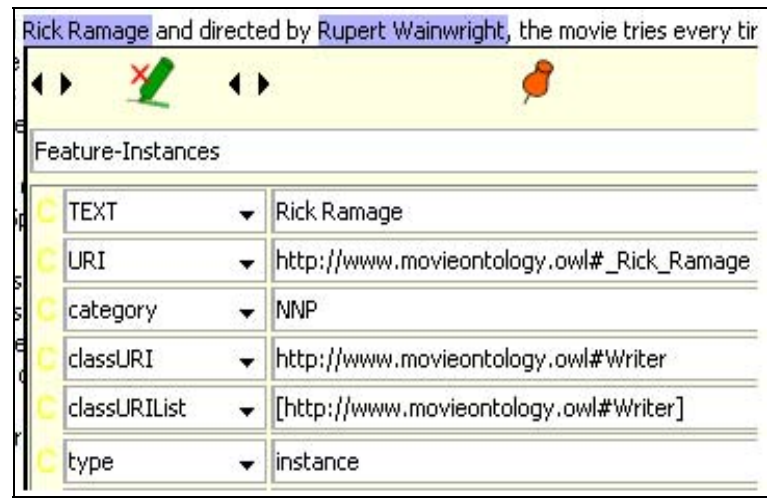

Figure 6: A snapshot of annotated feature by Onto Root Gazetteer 


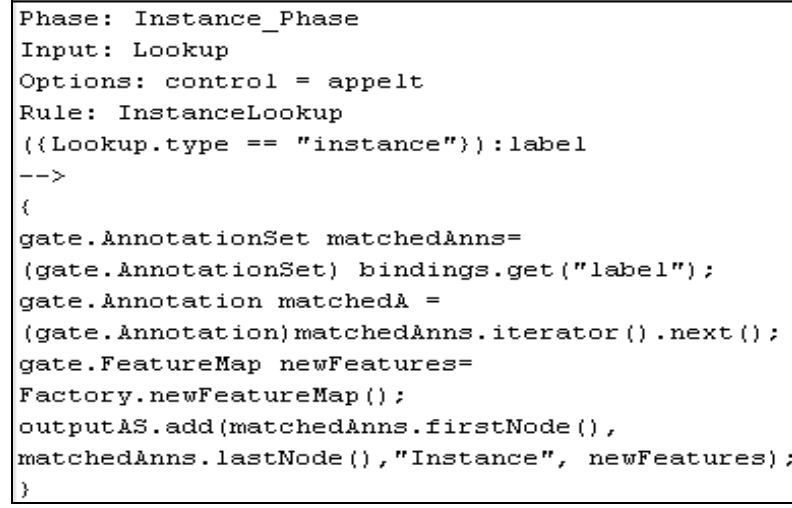

Figure 7: Example of Jape rules for instance annotation

instances were grouped under a set called "FeatureInstance". For that, we performed hand-crafted JAPE rules as shown in Fig. 7, which is "a finite state transducer that operates over annotations based on regular expressions" [15].

\section{Sentiment Matching}

In the opinion mining process, identifying sentiments within reviews is required in order to associate these with their corresponding features, and then calculating feature polarities. Our procedure for sentiment matching is based on utilising a lexicon of sentiment terms that are saved in GATE's Gazetteer which links between our sentiments and the root of each word in the pre-processed reviews.

\section{DISSCUSION \& CONCLUSION}

With the fast growth of World Wide Web 2.0, a great number of opinions about a variety of products have been published in blogs, forums, and social networks. Opinion mining tools are needed to enable users to efficiently process a large number of reviews found online, in order to determine the underlying opinions.

Some of the current Semantic Knowledge Based (SKB) approaches to opinion mining constructed an ontology based only on a domain's key concepts and their synonyms [18], whereas other SKB approaches enriched an ontology by adding more facts found in an Internet Movie Database [14]. This paper presents a new methodology for semantic modelling of the domain knowledge for opinion mining. In particular, the new methodology focuses on modelling the domain knowledge in such way that it can be translated to a formal ontology, which can then be automatically enriched with ground facts obtained from public Linked Open Data resources. The methodology also considers procedures to link between the formal ontology and Natural Language Processing. Our approach successfully enriches the ontology with the relevant ground facts. The ontology can then be used to perform a variety of data mining tasks including sentiment analysis and information retrieval.

In our future work, we aim to apply the proposed approach to improve semantic analysis and information retrieval tasks. We also aim to implement approaches to feature extraction and feature-sentiment association tasks using a semantic knowledge-based approach.

\section{REFERENCES}

[1] B. Agarwal, N. Mittal, P. Bansal and S. Garg, "Sentiment analysis using common-sense and context information," Computational Intelligence and Neuroscience, pp. 1-9, 2015.

[2] B. Agarwal, S. Poria, N. Mittal, A. Gelbukh and A. Hussain, "Concept-Level sentiment analysis with dependency-based semantic parsing: a novel approach," Cogn Comput, vol. 7, no. 4, pp. 487-499, 2015 .

[3] F. Ali, E. Kim and Y. Kim, "Type-2 fuzzy ontology-based opinion mining and information extraction: A proposal to automate the hotel reservation system," Applied Intelligence, vol. 42, no. 3, pp. 481-500, 2015 .

[4] F. Cruz, J. Troyano, F. Enríquez, F. Ortega and C. Vallejo, "Long autonomy or long delay? The importance of domain in opinion mining," Expert Systems with Applications, vol. 40, no. 8, pp. 3174 3184, 2013.

[5] B. Dalvi, E. Minkov, P. P. Talukdar, and W. W. Cohen, "Automatic gloss finding for a knowledge base using ontological constraints," in Proc. $8^{T H}$ ACM WSDM Int Conf. Web Search and Data Mining, 2015.

[6] Z. Deng, K. Luo and H. Yu, "A study of supervised term weighting scheme for sentiment analysis," Expert Systems with Applications, vol. 41, no. 7, pp. 3506-3513, 2014.

[7] M. Eirinaki, S. Pisal and J. Singh, "Feature-based opinion mining and ranking," Journal of Computer and System Sciences, vol. 78, no. 4, pp. 1175-1184, 2012.

[8] S. H. Ghorashi, R. Ibrahim, S. Noekhah and N. S. Dastjerdi, "A frequent pattern mining algorithm for feature extraction of customer reviews," International Journal of Computer Science Issues, vol. 9, no. 1, 2012.

[9] "Marl ontology specification," 2016. [Online]. Available: http://www.gsi.dit.upm.es/ontologies/marl/. [Accessed:28-Feb- 2016].

[10] M. Hu and B. Liu, "Mining and summarizing customer reviews," in Proc. 10th ACM SIGKDD Conf. Knowledge Discovery and Data Mining, ACM, 2004, pp. 168-177.

[11] B. Ma, D. Zhang, Z. Yan and T. Kim, "An LDA and synonym lexicon based approach to product feature extraction from online consumer product reviews,” J. Electron. Commer. Res., 14 (4), 2013, pp. 304-314.

[12] B. Matthews, "Semantic web technologies," E-learning., 6(6):8, 2005, p.3.

[13] Z. Sinobad, "Glossary of filmmographic terms," 2008.

[14] I. Peñalver-Martinez et al., "Feature-based opinion mining through ontologies," Expert Systems with Applications, vol. 41, no. 13, pp. 5995-6008, 2014

[15] "JAPE (linguistics)," Wikipedia, 2016. [Online]. Available: http:// en.wikipedia.org/wiki/JAPE_(linguistics). [Accessed: 05- Jan- 2016].

[16] "SPARQL," Wikipedia, 2016. [Online]. Available: http:// en.wikipedia.org/wiki/ SPARQL. [Accessed: 05- Jan- 2016].

[17] C. Yang, Z. Chen, T. Wang, and P. Sun, "Research on the Sentiment analysis of customer reviews based on the ontology of phone," in Proc. Int ICEMCT Conf. International Conference on Education, Management and Computing Technology, 2015.

[18] L. Zhao and C. Li, "Ontology based opinion mining for movie reviews," in Proc. $3^{\text {rd }}$ Int KSEM Conf. Knowledge Science, Engineering and Management, 2009, pp. 204- 214.

[19] L. Zhuang, F. Jing, and X.-Y. Zhu, "Movie review mining and summarization,"in Proc. CIKM Conf. 2006, pages 43-50. 